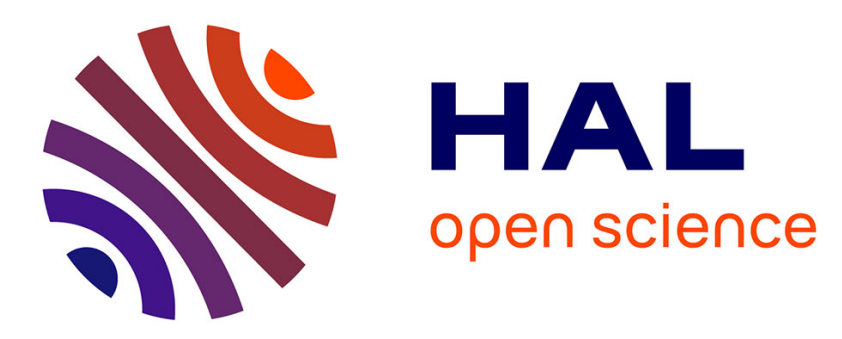

\title{
Numerical simulation of dip-coating in the evaporative regime
}

\author{
Mohar Dey, Frédéric Doumenc, Béatrice Guerrier
}

\section{To cite this version:}

Mohar Dey, Frédéric Doumenc, Béatrice Guerrier. Numerical simulation of dip-coating in the evaporative regime. European Physical Journal E: Soft matter and biological physics, 2016, 39 (2), pp.19. 10.1140/epje/i2016-16019-4 . hal-01284763

\section{HAL Id: hal-01284763 https://hal.sorbonne-universite.fr/hal-01284763}

Submitted on 8 Mar 2016

HAL is a multi-disciplinary open access archive for the deposit and dissemination of scientific research documents, whether they are published or not. The documents may come from teaching and research institutions in France or abroad, or from public or private research centers.
L'archive ouverte pluridisciplinaire HAL, est destinée au dépôt et à la diffusion de documents scientifiques de niveau recherche, publiés ou non, émanant des établissements d'enseignement et de recherche français ou étrangers, des laboratoires publics ou privés. 


\title{
Numerical simulation of dip-coating in the evaporative regime
}

\author{
Mohar Dey ${ }^{1}$, Frédéric Doumenc ${ }^{1,2}$, and Béatrice Guerrier ${ }^{1}$ \\ 1 Laboratoire FAST, Univ. Paris-Sud, CNRS, Université Paris-Saclay, F-91405, Orsay, France \\ 2 Sorbonne Universités, UPMC Univ Paris 06, UFR919, F-75005, Paris, France
}

Received: date / Revised version: date

\begin{abstract}
A hydrodynamic model is used for numerical simulations of a polymer solution in a dip-coatinglike experiment. We focus on the regime of small capillary numbers where the liquid flow is driven by evaporation, in contrast to the well-known Landau-Levich regime dominated by viscous forces. Lubrication approximation is used to describe the flow in the liquid phase. Evaporation in stagnant air is considered (diffusion-limited evaporation), which results in a coupling between liquid and gas phases. Self-patterning due to the solutal Marangoni effect is observed for some ranges of the control parameters. We first investigate the effect of evaporation rate on the deposit morphology. Then the role of the spatial variations in the evaporative flux on the wavelength and mean thickness of the dried deposit is ascertained, by comparing the 2D and 1D diffusion models for the gas phase. Finally, for the very low substrate velocities, we discuss the relative importance of diffusive and advective components of the polymer flux, and consequences on the choice of the boundary conditions.
\end{abstract}

PACS. 47.54.-r Pattern selection; pattern formation - 47.85.mb Coating flows - 47.55.np Contact lines

\section{Introduction}

Self patterning in drying processes with contact lines have been the focus of numerous experimental and theoretical studies during the last few years. Indeed, besides numerous industrial applications, configurations such as droplets or dip-coating processes involve complex physics due to multi-scale phenomena (see for instance [1-4]). In dipcoating like experiments, it is now well documented that several regimes appear depending on the capillary number $C a$, that compares capillary to viscous forces $(C a=$ $\eta V_{\text {sub }} / \gamma, \eta$ being the dynamic viscosity, $V_{\text {sub }}$ the substrate velocity and $\gamma$ the surface tension). We consider only small velocities for which inertial effects can be neglected. For sufficient capillary number, viscous forces are large enough to drag a film from the bath. This regime is known as the Landau-Levich regime $[5,6]$. The thickness of the dragged film is proportional to $\mathrm{Ca}^{2 / 3}$. More recently, studies have focused on the regime encountered for smaller capillary numbers, for which the liquid flow into the meniscus is mainly driven by the evaporation $[7,8]$. The present work deals with this evaporative regime, for the dip-coating like configuration depicted in figure 1 . We consider a receding meniscus on a moving substrate, fed by a rectangular inlet duct taking some polymer solution from an infinite and perfectly mixed reservoir at constant polymer volume fraction $\phi_{0}$. The bidimensional domain under consideration is composed of the inlet duct $\left(-L_{p}<x<0\right)$, followed by a liquid film $(0<x<W)$ surrounded by a

Send offprint requests to: Frédéric Doumenc gas domain $(0<x<W$ and $0<z<H)$. In contrast to the Landau-Levich regime, the dried film thickness is a decreasing function of the substrate velocity, since it is proportional to $V_{s u b}^{-1}$. This scaling may be derived by writing the mass balance for the solution and the solute [7,9-12], under the following assumptions: steady regime, no flow in the dry deposit, imposed concentration $\phi_{0}$ and negligible diffusive flux at the entrance of the duct (located at $x=-L_{p}$ in figure 1 ). The dry deposit thickness $h_{p}$ reads

$$
h_{p} \simeq \frac{F_{e v} \phi_{0}}{V_{s u b}}
$$

with $F_{e v}=\int_{0}^{W} v_{e v}(x) d x$ the total evaporation flux over the liquid free surface, where the solvent evaporates with the local rate $v_{e v}(x)$.

Moreover, for some values of the process parameters (substrate velocity, evaporation rate, reservoir solute concentration), the system may develop regular patterns induced by a periodic movement of the contact line. Despite the large number of experimental and theoretical studies published in the last decade, we are still lacking a complete understanding of the mechanisms responsible for this periodic regime (see Frastia et al. [13] for a complete review). Of particular interest are models predicting the periodic patterning without any artificial trigger. Such models were developed to describe the evaporation of dewetting liquid layers $[14,15,13]$, of droplets [16], or the transfer of a surfactant monolayer over a moving substrate [17]. 


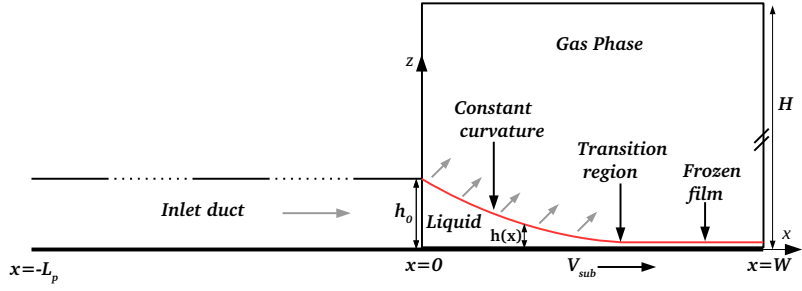

Fig. 1. Geometry of the dip-coating like configuration.

The present study follows a previous work dedicated to a dip-coating-like configuration [18]. A hydrodynamic model taking into account varying viscosity and Marangoni effect was developed and succeeded in capturing a selfpatterning phenomenon for some values of the substrate velocity. This same model is used to investigate two new points. First we focus on the the role of the evaporation rate on deposit morphology. In the configuration studied, solvent evaporates into ambient air. It is well known that evaporation flux close to a contact line or a meniscus exhibits strong spatial variations induced by $2 \mathrm{D}$ diffusion of the vapor in the gas phase. We address the importance of this spatial heterogeneity on the occurrence of the periodic regime by comparing a $2 \mathrm{D}$ diffusion model with a simplified 1D model.

In a second part, we discuss the relative importance of advection and diffusion on polymer transport. We show the importance of the inlet boundary condition at very low substrate velocity, where some unphysical results may be obtained due to backward diffusion polymer flow.

\section{Modeling}

This study examines a drying binary solution (one volatile solvent and one non-volatile solute) in a $2 \mathrm{D}$ meniscus. The geometry is represented in figure 1. The solution is in contact with a moving, flat substrate with a no-slip boundary condition. Gravity is neglected, and a uniform concentration over the thickness ( $\boldsymbol{z}$ direction) is assumed in the liquid phase. The free surface is in contact with air at atmospheric pressure and we assume a local thermodynamic equilibrium at the interface. The whole system is supposed to be at a constant temperature (isothermal problem). Evaporation is limited by the diffusion of solvent vapor into stagnant air. Two cases are considered for the evaporation flux: the vapor diffusion in the gas phase is described by a bidimensional or a one-dimensional model.

We focus on the long-term behavior, when a steady state or a periodic regime has been reached, the latter case corresponding to self-patterning of the deposit.

\subsection{Hydrodynamics in the film: lubrication approximation}

The lubrication approximation is used to describe the liquid flow in the film $(0<x<W$ in figure 1$)$. Previous simulations show that this domain may be divided into three parts schematically depicted in figure 1 [18]. The first part of the meniscus corresponds to the static solution at equilibrium, with constant curvature. At the opposite, close to the end of the simulation domain, the film is gelled due to the very high viscosity and is simply translated at the substrate velocity. In between is the transition region, with varying curvature and strong concentration gradients.

The direction parallel to the substrate (flow main direction) is denoted by $\boldsymbol{x}$; the axis normal to the substrate is denoted by $\boldsymbol{z}$. Assuming a Newtonian fluid, Stokes equations with small slope approximation lead to the following standard result [19]:

$$
Q(x, t)=V_{s u b} h-\frac{h^{3}}{3 \eta(\phi)} \frac{\partial P}{\partial x}+\frac{h^{2}}{2 \eta(\phi)} \frac{d \gamma}{d \phi} \frac{\partial \phi}{\partial x},
$$

where $V_{\text {sub }}$ is the substrate velocity, $h$ is the liquid height, $P$ is the liquid pressure, $\eta$ is the dynamic viscosity, $\gamma$ is the surface tension, and $\phi$ is the polymer volume fraction. The three terms on the right-hand side of equation (2) describe the flow produced by the substrate motion, the pressure gradient and the surface tension gradient (solutal Marangoni effect), respectively. The surface tension is assumed to vary linearly with the polymer volume fraction (non linear effect that may occur in polymer solutions [20] are neglected).

The pressure drop across the free surface includes the capillary pressure and the disjoining pressure and reads

$$
P-P_{a t m}=-\gamma(\phi) \frac{\partial^{2} h}{\partial x^{2}}-A /\left(6 \pi h^{3}\right),
$$

with $A$ the Hamaker constant. The expression of the disjoining pressure accounts for long range van der Waals interactions and corresponds to a complete wetting configuration $[21,22]$. The disjoining pressure impacts significantly on the results for very thin films only. This is not the case for most of the configurations considered in the following, except for some specific cases considered in section 4.2 .

$Q(x, t)$ is the liquid volumic flux (by unit of width) at abscissa $x$ and time $t$ over a cross-section normal to $\boldsymbol{x}$,

$$
Q(x, t)=\int_{0}^{h(x)} u(x, z) d z
$$

where $u(x, z)$ is the solution velocity component in the $\boldsymbol{x}$ direction.

The flux of non-volatile solute $Q_{p}$ reads

$$
Q_{p}=\phi Q-D h \frac{\partial \phi}{\partial x}
$$

The second term on the right-hand side of equation (5) is the Fick's law integrated over the film thickness, which accounts for polymer/solvent relative motion. $D$ is the mutual diffusion coefficient of the binary system. For polymer solutions, $D$ keeps the same order of magnitude for polymer volume fraction less than about 0.7 , and then 
decreases strongly (several orders of magnitude) with increasing polymer concentration (cf. for instance [23]). In the present model $D$ is assumed constant. Indeed previous simulations have shown that the fall of $D$ for $\phi>0.7$ did not change the results significantly. A more sophisticated approach would consist to compute $D$ from the expression of the osmotic pressure, as done by U.Thiele and co-authors [24].

The global mass balance and the non-volatile solute mass balance respectively read

$$
\begin{aligned}
\frac{\partial h}{\partial t}+\frac{\partial Q}{\partial x} & =-v_{e v}(x, t), \\
\frac{\partial(\phi h)}{\partial t}+\frac{\partial Q_{p}}{\partial x} & =0,
\end{aligned}
$$

where the local evaporation velocity $v_{e v}(x, t)$ is positive for evaporation.

\subsection{Hydrodynamic boundary conditions}

At $x=0$ we impose the liquid height $h_{0}$ and the curvature $C_{0}$. The height $h_{0}$ is supposed to be small enough to ensure the validity of a small slope approximation. Imposing $h_{0}$ and $C_{0}$ at $x=0$ creates a meniscus section of static length $L_{s t}=\sqrt{2 h_{0} / C_{0}}$. $\left(L_{s t}\right.$ corresponds to the intersection of a parabolic meniscus with the horizontal axis with zero contact angle). Variations of curvature and height at $x=0$, produced by the evaporation or the substrate motion, are supposed to be negligible. The hydrodynamic boundary conditions at $x=0$ are then

$$
h(x=0)=h_{0}, \frac{\partial^{2} h}{\partial x^{2}}(x=0)=C_{0} .
$$

Two different solutal boundary conditions are compared. The first one is similar to the one used in our previous study [18], i.e. a constant value of the polymer volume fraction is imposed at $x=0$,

$$
\mathrm{CL}_{\text {inlet } 1}: \quad \phi(x=0)=\phi_{0} .
$$

However, in experiments such as the ones described in $[9,10]$, the polymer volume fraction is not imposed at the meniscus, but at some distance upstream. Thus a more realistic boundary condition has also been considered. A long inlet duct of length $L_{p}$ and constant height $h_{0}$ is introduced upstream of the meniscus, with $L_{p}>>W$. The reservoir concentration is now imposed at the entrance of the inlet duct,

$$
\mathrm{CL}_{\text {inlet } 2}: \quad \phi\left(x=-L_{p}\right)=\phi_{0} .
$$

There is no free surface and thus no evaporation in this inlet duct. The solute distribution is thus described by an advection-diffusion equation:

$$
\text { for }-L_{p}<x<0: \quad \frac{\partial \phi}{\partial t}+\frac{Q_{0}}{h_{0}} \frac{\partial \phi}{\partial x}=\frac{\partial}{\partial x}\left(D \frac{\partial \phi}{\partial x}\right),
$$

with $Q_{0}=Q(x=0)$.
Close to $x=W$, the deposit is assumed frozen. We thus impose the condition

$$
\text { for } x \geq W: \quad \frac{\partial h}{\partial t}+V_{s u b} \frac{\partial h}{\partial x}=0 .
$$

\subsection{Gas phase and evaporation at the free surface}

\subsection{1 $\mathcal{M}_{G 2 D}: 2 \mathrm{D}$ diffusion in the gas phase}

The local evaporation velocity $v_{e v}(x, t)$ in equation (6) is calculated by solving a diffusion problem in the gas phase for a domain of length $W$ and height $H$ ( $H$ would roughly correspond to the boundary layer thickness for a process with a forced air-flow above the free surface). Because $H$ is considerably larger than $h_{0}$, we assume a rectangular domain for the gas phase. The liquid thickness variations are therefore neglected in the computation of the mass transfer in the gas phase. The bottom boundary of this domain corresponding to the liquid-gas interface is thus: $z=0$ and $0<x<W$.

Two models are compared in the following. In the first one, denoted $\mathcal{M}_{G 2 D}$, a $2 \mathrm{D}$ diffusion equation is solved in the gas phase. The solvent vapor is assumed to diffuse into stagnant air and the diffusion equation in the gas phase reads

$\frac{\partial c_{g}}{\partial t}=D_{g}\left(\frac{\partial^{2} c_{g}}{\partial x^{2}}+\frac{\partial^{2} c_{g}}{\partial z^{2}}\right)$, for $0<x<W$ and $0<z<H$,

where $c_{g}$ is the solvent vapor concentration in the air, and $D_{g}$ is the vapor diffusion coefficient. The vertical walls are assumed to be impermeable. A Dirichlet condition with a zero solvent vapor concentration is imposed at the top. We thus obtain the following boundary conditions for the gas domain (see figure 1),

$$
\begin{gathered}
\frac{\partial c_{g}}{\partial x}=0 \text { for }(x=0 \text { or } x=W) \text { and } 0<z<H \\
c_{g}=c_{\infty} \text { for } 0 \leq x \leq W \text { and } z=H
\end{gathered}
$$

Another boundary condition corresponding to the coupling between the liquid and the gas phases is obtained from the local thermodynamic equilibrium at the free surface.

Assuming that the vapor behaves as an ideal gas, the vapor chemical potential reads

$$
\mu_{v}=\mu_{v 0}+R T \ln \frac{P_{v s}}{P_{v s 0}},
$$

where $R$ is the ideal gas constant, $T$ is the temperature, $P_{v s}$ is the saturating vapor pressure over the solution at pressure $P, P_{v s 0}$ is the saturating vapor pressure over the pure solvent at pressure $P_{a t m}$, and $\mu_{v 0}$ is the vapor chemical potential when $P_{v s}=P_{v s 0}$.

The solvent chemical potential in the incompressible liquid phase reads

$$
\mu_{S}=\mu_{s 0}+\bar{v}_{s}\left[\left(P-P_{a t m}\right)-\Pi\right]
$$


where $\Pi$ is the osmotic pressure, $\bar{v}_{s}$ is the solvent molar volume, and $\mu_{s 0}$ is the chemical potential of the pure solvent when $P=P_{\text {atm }}$.

Using equations (16-17) along with the equation (3), the ideal gas law and the standard relation $\bar{v}_{s} \Pi=-R T \ln (a)$ where $a$ is the solvent activity, the thermodynamic equilibrium at the interface reads

$$
c_{g}=a(\phi, T) P_{v s 0}(T) \frac{M_{s}}{R T} \exp \left[-\frac{\bar{v}_{s}}{R T}\left(\gamma \frac{\partial^{2} h}{\partial x^{2}}+\frac{A}{6 \pi h^{3}}\right)\right],
$$

$$
\text { for } 0 \leq x \leq W \text { and } z=0 \text {, }
$$

where $M_{s}$ is the solvent molar mass. The solvent activity is given by the classical Flory-Huggins theory [25]. For a polymer of high molar mass, $a=(1-\phi) \exp \left(\phi+\chi \phi^{2}\right)$ where $\chi$ is the polymer-solvent interaction parameter.

Regarding the evaporation flux, it is worthwhile to note the difference between a polymer solution and a pure fluid, as considered for instance by Eggers and Pismen [26]. Indeed, unlike for pure fluid, the variation of solvent activity with concentration allows to regularize the problem for most of the configurations studied, without the need of disjoining pressure. It is only for some configurations where a very thin liquid film $(\sim 1 \mathrm{~nm})$ is obtained that disjoining pressure must be considered (see section $4.2)$.

The last equation represents the mass flux conservation across the interface:

$$
\rho v_{e v}=-D_{g} \frac{\partial c_{g}}{\partial z}
$$

where $\rho$ is the pure solvent density.

\subsection{2 $\mathcal{M}_{G 1 D}: 1 \mathrm{D}$ diffusion in the gas phase}

In the second model, denoted $\mathcal{M}_{G 1 D}$ in the following, we get the evaporation flux from the phenomenological expression

$$
\rho v_{e v}=h_{m}\left(c_{g}(z=0)-c_{\infty}\right),
$$

where $h_{m}$ is a constant mass transfer coefficient.

The relation (20) corresponds to $1 \mathrm{D}$ and steady transfer in the gas phase $\left(\partial^{2} c_{g} / \partial z^{2}=0\right)$. With these assumptions, $h_{m}$ is related to the boundary layer thickness $\delta_{g}$ by the relation $h_{m}=D_{g} / \delta_{g}$. Notice that another interpretation of the relation (20) would be possible in the different configuration of an atmosphere of pure solvent (no inert gas). In that case, evaporation would be limited by kinetic effects, and the evaporation rate would be given by the relation (20), with $h_{m}$ computed from the Hertz-Knudsen theory [27].

\section{Resolution}

A part of the results have been obtained by solving the above equations with a homemade Fortran software (finite differences with pure implicit scheme of order 1 in time and 2 in space [28]), and the other part with Comsol Multiphysics, a commercial finite element software (BDF solver, quadratic Lagrange elements). Both softwares give the same results, with a lower computational time in the ) latter case. The first run is performed with an arbitrary initial condition. Then the evaporation flux is changed stepwise and the solution obtained for the previous step is used as initial condition. The effect of the initial conditions on the long time behavior has been analyzed in a previous study and was shown to have a negligible impact on the results. (cf. [18] for more details).

Numerical solutions provide the liquid film shape, the local evaporation flux, the concentration field in the gas (for $\mathcal{M}_{G 2 D}$ ), and the concentration and velocity fields in the solution. The following geometrical values are used: $H=3 \mathrm{~mm}, W=1 \mathrm{~mm}, C_{0}=2 \mathrm{~mm}^{-1}, h_{0}=0.1 \mathrm{~mm}$, and $L_{p}=10 \mathrm{~mm}$ for the boundary condition $\mathrm{CL}_{\text {inlet } 2}$. At $z=$ $H$ the Dirichlet condition for concentration is $c_{\infty}=0$.

Physico-chemical properties are those of Polyisobutylene (PIB)/toluene [29-31]. The toluene molar mass and diffusion coefficient in the gas phase are $M_{s}=92.14 \mathrm{~g} \cdot \mathrm{mol}^{-1}$, and $D_{g}=8.6 \times 10^{-6} \mathrm{~m}^{2} \cdot \mathrm{s}^{-1}$, respectively. The solution density and the diffusion coefficient are assumed to be constant: $\rho=900 \mathrm{~kg} \cdot \mathrm{m}^{-3}$ and $D=10^{-10} \mathrm{~m}^{2} \cdot \mathrm{s}^{-1}$. The surface tension $\gamma$ is assumed to increase linearly with the solute volume fraction from $\gamma=28 \times 10^{-3} \mathrm{~N} . \mathrm{m}^{-1}$ (pure toluene) to $\gamma=34 \times 10^{-3} \mathrm{~N} . \mathrm{m}^{-1}$ (pure PIB). The viscosity $\eta$ exhibits a strong increase with the solute volume fraction (12 orders of magnitude from pure toluene to pure PIB for a PIB molar mass of $\left.M_{W}=500 \mathrm{~kg} \cdot \mathrm{mol}^{-1}\right)$. The following empirical law gives $\eta$ in Pa.s at $T=25^{\circ} \mathrm{C}: Y=8.22+$ $13.2 X+5.2 X^{2}+0.70 X^{3}$, where $Y=\log _{10}(\eta)$ and $X=$ $\log _{10}(\phi)$. The saturated vapor pressure is $P_{v s 0}=3792 \mathrm{~Pa}$ at $T=25^{\circ} \mathrm{C}$. The interaction Flory parameter $\chi$ has been measured by gravimetric experiments, $\chi=0.45+0.30 \phi$ at $T=25^{\circ} \mathrm{C}$. The Hamaker constant $A$ is $10^{-19} \mathrm{~J}$. Temperature is $25^{\circ} \mathrm{C}$ unless specified.

Although the results are presented in dimensional variables, we present here the main dimensionless parameters involved in this problem. We use $h_{0}$ and $\sqrt{h_{0} / C_{0}}$ for the vertical and horizontal scales, respectively, $V_{\text {sub }}$ for the velocity scale, $\eta_{0}=\eta\left(\phi_{0}\right)$ and $\gamma_{0}=\gamma\left(\phi_{0}\right)$ for the viscosity and surface tension scales. From the lubrication equation (2) we get the modified Capillary number $\delta$ and Marangoni number $M a$ :

$$
\begin{gathered}
\delta=\frac{3 \eta_{0} V_{\text {sub }}}{\gamma_{0}\left(h_{0} C_{0}\right)^{3 / 2}}, \\
M a=\frac{\gamma_{\text {solute }}-\gamma_{\text {solvent }}}{2 \eta_{0} V_{\text {sub }}}\left(h_{0} C_{0}\right)^{1 / 2} .
\end{gathered}
$$

The Peclet number is obtained from the equation (5),

$$
P e=\frac{V_{s u b}}{D}\left(h_{0} / C_{0}\right)^{1 / 2}
$$

For most of the simulations, we have

$6 \times 10^{-6} \lesssim \delta \lesssim 4 \times 10^{-4}, 4 \times 10^{3} \lesssim M a \lesssim 3 \times 10^{5}, 10 \lesssim P e \lesssim 70$.

An exception is figure 11, where the substrate velocity is varied over a large number of decades. 


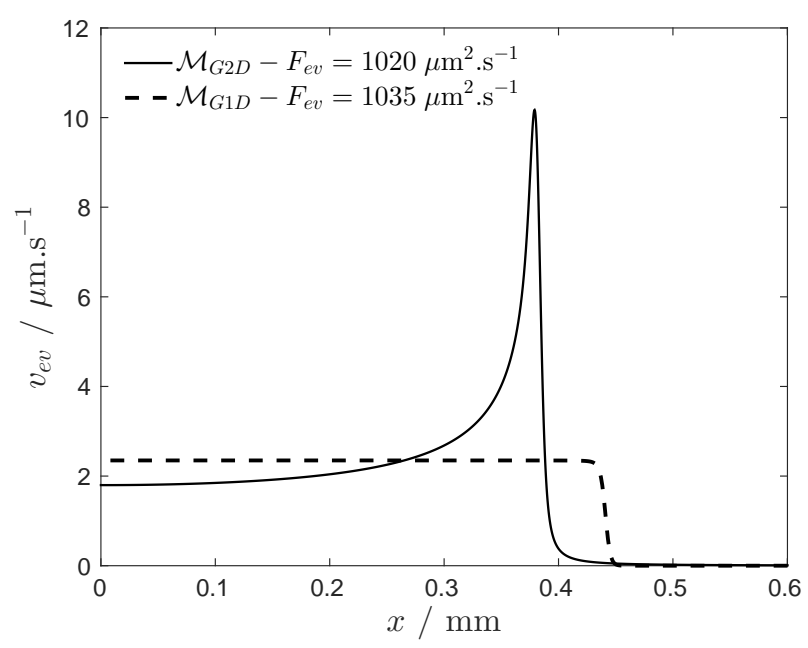

Fig. 2. Local evaporation rate for $\mathcal{M}_{G 1 D}$ and $\mathcal{M}_{G 2 D}$ models and two close values of $F_{\text {ev }}\left(\phi_{0}=0.01\right.$ and $\left.V_{\text {sub }}=30 \mu \mathrm{m} / \mathrm{s}\right)$.

\section{Results and discussion}

\subsection{Evaporation flux}

In this section we explore the role of the evaporation rate on the deposit morphology. Results are presented as a function of the global evaporation flux $F_{e v}=\int_{0}^{W} v_{e v}(x) d x$. Note that $F_{e v}$ is not a direct input of the model. In the $2 \mathrm{D}$ model $\left(\mathcal{M}_{G 2 D}\right)$, it is changed by modifying the saturated vapor pressure $P_{v s 0}(T)$ in equation (18). To focus on evaporation rate only, all other properties are still taken at $T=25^{\circ} \mathrm{C}$. For $\mathcal{M}_{G 1 D}$, the evaporation rate is changed by modifying the mass transfer coefficient $h_{m}$ (cf. equation $(20))$.

It is well known that the evaporation flux close to a contact line or a meniscus exhibits strong spatial variations induced by $2 \mathrm{D}$ diffusion effect in the gas phase. Using electrostatic analogy, Deegan and co-authors derived a simplified expression of the evaporative flux that diverges at the contact line [32]. The model used here for an evaporating solution suppresses the divergence, but a significant peak in the profile of the evaporation rate still remains, as illustrated in figure 2 (continuous line).

First we focus on evaporation flux intensity, using $\mathcal{M}_{G 2 D}$ and varying $F_{e v}$. Then we analyze the impact of flux spatial inhomogeneity by comparing 1D and 2D models in the gas $\left(\mathcal{M}_{G 1 D}\right.$ and $\left.\mathcal{M}_{G 2 D}\right)$. Simulations are done with the boundary condition $C L_{\text {inlet } 1}$ and disregarding disjoining pressure in the lubrication equation. These two assumptions are valid as the substrate velocity is high $\left(V_{s u b}=\right.$ $30 \mu \mathrm{m} / \mathrm{s}$, see next section) and the obtained film thickness is large enough to disregard disjoining pressure.

\subsection{1 $\mathcal{M}_{G 2 D}$ - Effect of evaporation flux intensity}

The substrate velocity is fixed at $V_{s u b}=30 \mu \mathrm{m} / \mathrm{s}$, and the control parameters are the evaporation flux $F_{e v}$ and the bulk solute concentration $\phi_{0}$. Different behaviors may be observed, depending on these control parameters. Typical liquid film profiles are illustrated in semi-log scale in figure 3 for $\phi_{0}=0.01$ and three values of $F_{e v}$. All the profiles first overlap with the constant curvature meniscus and then enter the transition region, earlier for the highest value of the evaporation flux. For $F_{e v}=1720 \mu \mathrm{m}^{2} / \mathrm{s}$ we obtain a steady solution, with a monotonic profile. The intermediate value of $F_{e v}$ also leads to a steady solution, but with a bump in the transition region. At last, for $F_{e v}=$ $400 \mu \mathrm{m}^{2} / \mathrm{s}$ no stationary solution is found. After formation, the bump moves away from the meniscus, and dries out with a strong increase of its viscosity, which prevents the possibility of a flow in the substrate framework. The bump is then advected to the outlet by the substrate motion. A new bump appears and grows in the transition region, and follows the same way (notice that the red curve in figure 3 is thus a "snapshot" of the liquid film at a given time). The formation of a bump by a solutal Marangoni flow has already been observed and discussed in the literature (see for instance [33] and [34] and the discussion in [18]). The periodic regime and the resulting deposit self-patterning are thus a consequence of the solutal Marangoni effect. Indeed, in the present model, a constant surface tension always results in a monotonic meniscus profile (no bump), and a steady state at long time (flat deposit).

The morphological phase diagram as a function of control parameters is shown in figure 4. It exhibits the different behaviors of the meniscus described above: steady solutions with or without formation of a bump or periodic behavior. The periodic sub-regime is only obtained for low bulk concentrations $\left(\phi_{0}=0.01\right.$ and 0.02$)$ and for a small interval of evaporation flux (red circles in figure 4). Qualitatively, the morphological phase diagram and meniscus typical shapes are similar to those obtained when varying the substrate velocity [18]. The effect of $F_{e v}$ and $V_{s u b}$ are thus qualitatively comparable.

As already said, after the transition region the system is frozen due to the very high viscosity of the solution. The pattern is thus simply translated at the substrate velocity $V_{s u b}$. It is thus possible to estimate the wavelength and amplitude of the deposit. Typical morphologies of the deposits are given in figure 5 for $\phi_{0}=0.01$. For ease of comparison, two wavelengths are drawn for each value of $F_{e v}$, and the local amplitude is scaled by the mean dry thickness. At low evaporation flux the periodic structure shows a main peak as well as a small secondary peak. This structure evolves towards a reverse morphology, with a single valley, as the evaporation flux decreases. The mean thickness of the dry film is proportional to $F_{e v}$, which is the scaling expected from the simplified model (1).

The evolution of the wavelength as a function of the evaporation flux is shown in figure 6 (red circles for model $\left.\mathcal{M}_{G 2 D}\right)$. At high evaporation flux we observe a strong increase of the wavelength, which corresponds to the transition from the periodic regime to the steady one when $F_{e v}$ exceeds a given value. Qualitatively we observe the same transition when the control parameter is the substrate velocity, the divergence of the wavelength being observed this time when $V_{\text {sub }}$ is smaller than a given value. This 


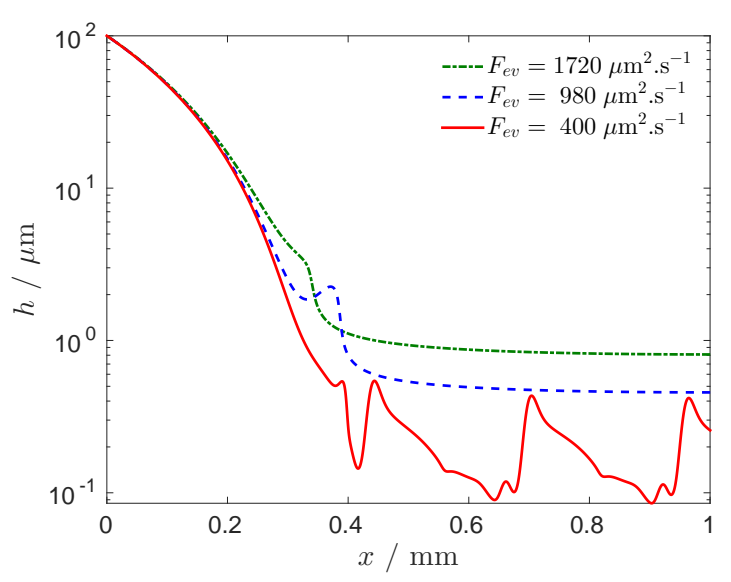

Fig. 3. Liquid height for different evaporation flux $\left(\phi_{0}=0.01\right.$ and $V_{\text {sub }}=30 \mu \mathrm{m} / \mathrm{s}$ ). From top to bottom: $F_{\text {ev }}=1720 \mu \mathrm{m}^{2} / \mathrm{s}$ (steady sub-regime, monotonic profile); $F_{e v}=980 \mu \mathrm{m}^{2} / \mathrm{s}$ (steady sub-regime, stationary bump); $F_{e v}=400 \mu \mathrm{m}^{2} / \mathrm{s}$ (periodic sub-regime, moving bump)

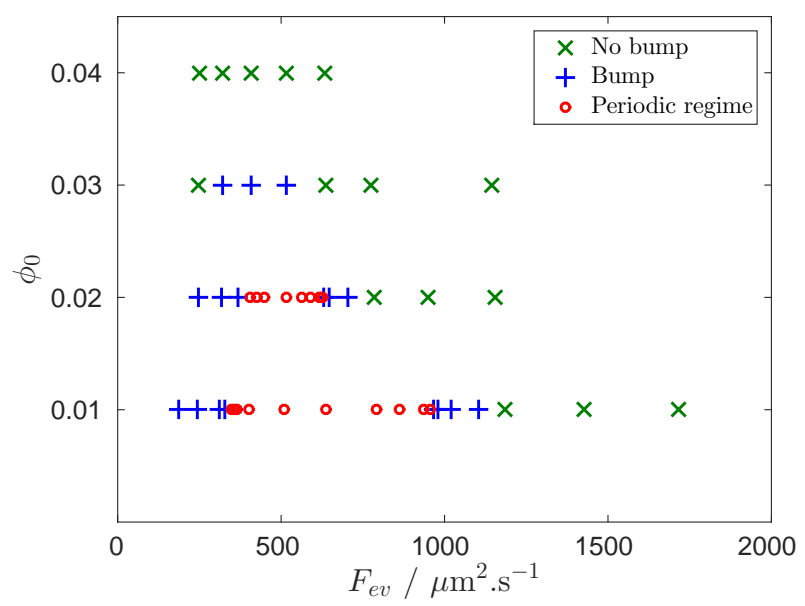

Fig. 4. Morphological phase diagram of meniscus shapes, in the plane spanned by the evaporation flux $F_{\text {ev }}$ and the bulk polymer volume fraction $\phi_{0}\left(V_{s u b}=30 \mu \mathrm{m} / \mathrm{s}\right)$.

strong increase in wavelength indicates a global bifurcation as also hypothesized in [13] and shown for another line-deposition mechanism in [35].

\subsubsection{Comparison of $\mathcal{M}_{G 1 D}$ and $\mathcal{M}_{G 2 D}$}

To infer the role of spatial variation of the evaporation rate induced by $2 \mathrm{D}$ diffusion in the gas phase, comparison is made between 2D and 1D models. For the same global evaporation flux $F_{e v}$, figure 2 illustrates typical profiles of the evaporation rate for models $\mathcal{M}_{G 2 D}$ and $\mathcal{M}_{G 1 D}$. Unlike the peak induced by $2 \mathrm{D}$ effects, $\mathcal{M}_{G 1 D}$ leads to a constant evaporation rate. Indeed, for polymer solutions, the solvent activity is close to one for polymer volume fraction smaller than about 0.6 . It is only when the solute concentration increases sufficiently at the end of the meniscus (for $x>0.42$ ) that the evaporation rate falls down, due to the solvent activity decrease.

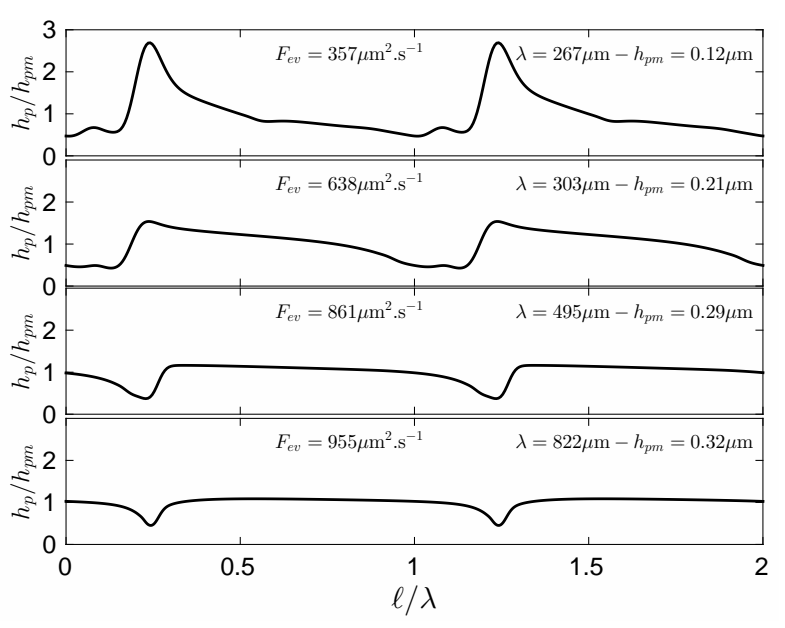

(a) $\mathcal{M}_{G 2 D}$

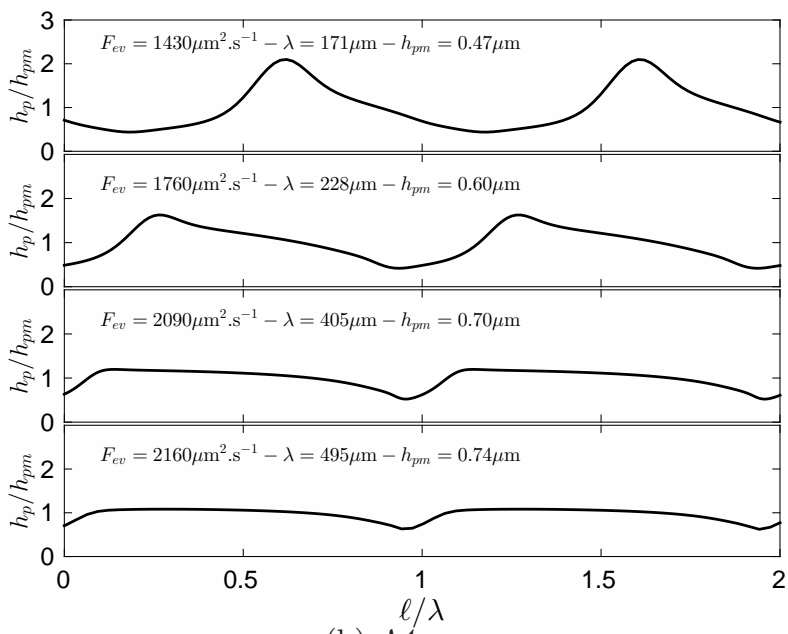

(b) $\mathcal{M}_{G 1 D}$

Fig. 5. Deposit height for the periodic sub-regime $\left(\phi_{0}=0.01\right.$ and $V_{\text {sub }}=30 \mu \mathrm{m} / \mathrm{s}$ ), for $\mathcal{M}_{G 2 D}$ model (top) and $\mathcal{M}_{G 1 D}$ (bottom). Abscissa and ordinate are scaled by the wavelength $\lambda$ and mean thickness $h_{p m}$, respectively.

All other things being equal, a periodic sub-regime is still observed for the $\mathcal{M}_{G 1 D}$ model, but for higher values of the global evaporation flux $F_{e v}$, as illustrated in figure 6. Wavelengths are of the same order than those obtained with $\mathcal{M}_{G 2 D}$. Deposit morphologies are also rather similar, although topographies are a little smoother with $\mathcal{M}_{G 1 D}$ (figure 5). As a conclusion, the peak of the evaporation rate characteristic of $2 \mathrm{D}$ diffusion in the gas phase is not necessarily required to get the instability (this is in agreement with several studies in the literature, see for instance [13]). The periodic regime could also be observed for $1 \mathrm{D}$ diffusion but in a different range of evaporation flux.

\subsection{Polymer transport: diffusion against advection}

We see from equation (5) that the polymer flux $Q_{p}$ is the sum of an advection term $Q_{p-a d}=\phi Q$ and a diffu- 


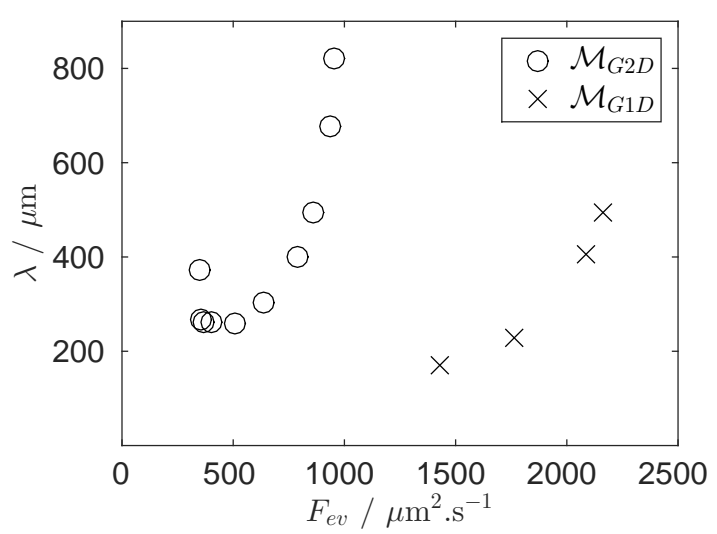

Fig. 6. Pattern wavelength in the periodic sub-regime for $\mathcal{M}_{G 1 D}$ and $\mathcal{M}_{G 2 D}$ model $\left(\phi_{0}=0.01\right.$ and $\left.V_{\text {sub }}=30 \mu \mathrm{m} / \mathrm{s}\right)$.

sion term $Q_{p-d i f}=-D h \partial \phi / \partial x$. In this section, we investigate the relative importance of these two terms, and the consequences of diffusion on the choice of the solutal boundary condition at the system entrance. Simulations are performed with $\mathcal{M}_{G 2 D}\left(T=25^{\circ} \mathrm{C}\right)$ and $\phi_{0}=0.01$ Results are presented for $V_{\text {sub }}=5 \mu \mathrm{m} / \mathrm{s}$ and $10 \mu \mathrm{m} / \mathrm{s}$, and boundary conditions $\mathrm{CL}_{\text {inlet } 1}$ (no inlet duct) and $\mathrm{CL}_{\text {inlet } 2}$ (inlet duct of length $L_{p}=1 \mathrm{~cm}$ ). For these values of the control parameters, we get a steady state at long time. From equation (7) it follows that $Q_{p}$ is constant along the whole domain.

Figures 7 and 8 show the polymer volume fraction $\phi(x)$ and the liquid height $h(x)$, respectively. Typically, $\phi(x)$ increases sharply from the bulk value $\phi_{0}$ to high concentration. For the higher velocity $V_{s u b}=10 \mu \mathrm{m} / \mathrm{s}$, the front occurs far from the entrance for boundary conditions $C L_{\text {inlet } 1}$ or $C L_{\text {inlet } 2 .}$. Diffusion can thus be neglected over advection at $x=0$ (figure 9 ), and both boundary conditions give very close results (see figures 7 and 8).

Things are very different for the lower velocity $V_{\text {sub }}=$ $5 \mu \mathrm{m} / \mathrm{s}$. Using $C L_{\text {inlet } 2}$, it can be observed that the concentration front enters into the inlet duct (figure 7) and thus diffusion flux is no more negligible at $x=0$ (figure 10). However, the inlet duct is long enough to recover a negligible diffusion flux at the domain entrance located at $x=-L_{p}$. But if $\phi=\phi_{0}$ is imposed at $x=0$, the simulation converges towards a strongly different solution. Indeed, $Q_{p-a d}$ and $Q_{p-d i f}$ compensate each other, and $Q_{p}$ is almost zero (figure 10). This very small polymer flux results in a very thin deposit, of nanometric thickness determined by the disjoining pressure term in equation (18) (figure 8).

A consequence of the importance of diffusion at the domain entrance is shown in figure 11, where the dried deposit thickness is plotted as a function of the substrate velocity, along with the prediction of the simplified model (1). When the boundary condition $\mathrm{CL}_{\text {inlet } 2}$ is used, the deposit thickness follows the law (1) in the whole range of velocity. Conversely, the boundary condition $\mathrm{CL}_{\text {inlet } 1}$ leads to a kind of dewetting transition for velocities $V_{\text {sub }} \leq$ $5 \mu \mathrm{m} / \mathrm{s}$, where diffusion almost balances advection. This results in a strong departure from the model (1), con-

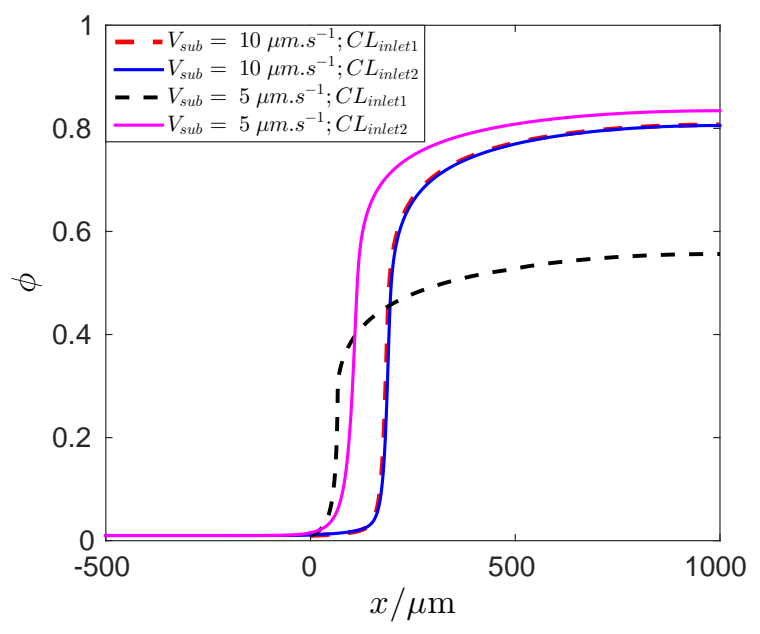

Fig. 7. Concentration variation in the meniscus. Solid lines correspond to the boundary condition $C L_{\text {inlet } 2}$ and dashed lines to the boundary condition $C L_{\text {inlet } 1}\left(\phi_{0}=0.01\right.$ and $\mathcal{M}_{G 2 D}$ with $\left.T=25^{\circ} \mathrm{C}\right)$.

sistent with the fact that this model has been derived assuming negligible diffusion. Such a dewetting has not been observed in experiments described in $[9,10]$. Therefore, it may be necessary to consider an inlet duct to reproduce the experimental conditions with the model.

\section{Conclusion}

The effect of the evaporation flux on the deposit characteristics have been investigated. We show that the evaporation intensity can be used to control the deposit characteristics, i.e. the mean thickness, the wavelength and the shape. Modifying the spatial variations of the local evaporation velocity $v_{e v}(x)$ shifts the self-patterning domain to other values of the total flux $F_{e v}$, but results are qualitatively similar.

In the second part, we show after discussing the relative importance of diffusion and advection that the length of the zone preceding the meniscus, introduced in $C L_{\text {inlet } 2}$ is of major importance at very low substrate velocity. We have compared two extreme cases. In the first one, the inlet duct is long enough to ensure quasi null polymer diffusion at the entrance of the duct $\left(x=-L_{p}\right)$. In the second one, we impose the polymer volume fraction at $x=0$ (i.e. $C L_{\text {inlet } 1}$ ), and a dewetting transition is obtained at low velocity. Some intermediate cases should be investigated. Beyond the geometry studied, these results show that regime of very low contact line velocities may be strongly impacted by diffusion. We are currently performing experiments on polymer solutions in a dip-coating like configuration to test the approach presented in this paper.

Acknowledgments: The authors thank ANR EVAPEC 13-BS09-0010-01 for funding. 


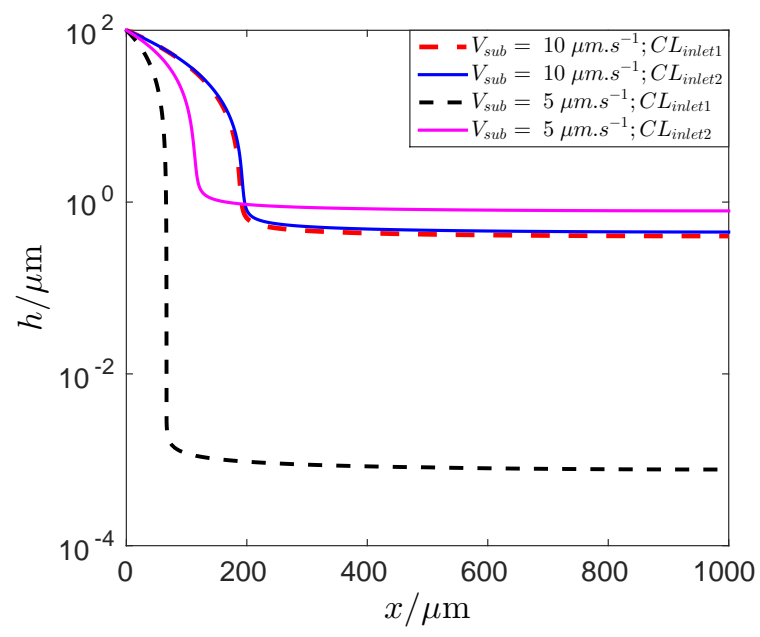

Fig. 8. Meniscus profile (semi-log scale). Solid lines correspond to the boundary condition $C L_{\text {inlet } 2}$ and dashed lines to the boundary condition $C L_{\text {inlet } 1}\left(\phi_{0}=0.01\right.$ and $\mathcal{M}_{G 2 D}$ with $T=$ $\left.25^{\circ} \mathrm{C}\right)$.

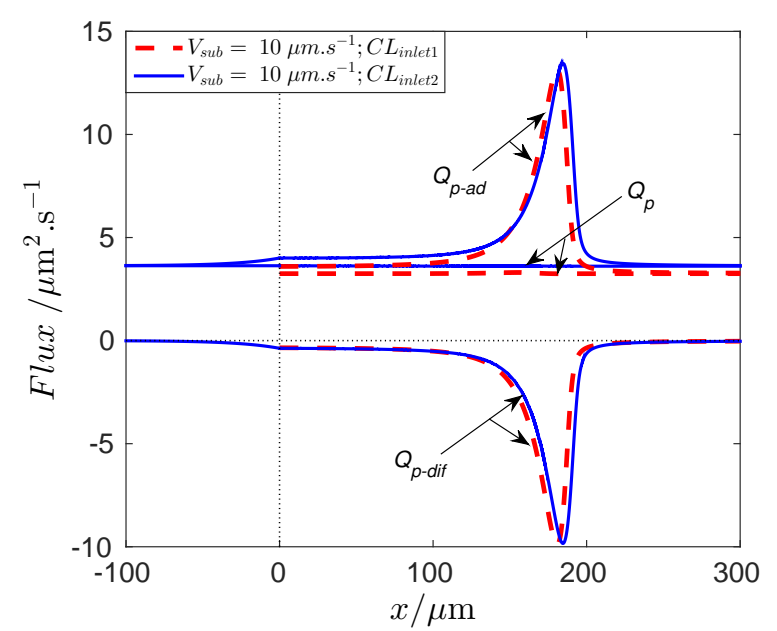

Fig. 9. Diffusion and advection components of the polymer flux for $V_{\text {sub }}=10 \mu \mathrm{m} / \mathrm{s}\left(\phi_{0}=0.01, \mathcal{M}_{G 2 D}\right.$ with $T=25^{\circ} \mathrm{C}$, zoom in the range $-100 \mu \mathrm{m} \leq x \leq 300 \mu \mathrm{m})$.

\section{References}

1. W. Han, Z. Lin., Angew. Chem. Int. Ed., 51, (2012), 15341546.

2. R.G. Larson, Aiche J., 60 (2014) 1538-1571.

3. U. Thiele, Adv. Colloid Interface Sci., 206 (2014) 399-413.

4. L. Li, M.H. Köpf, S.V. Gurevich, R. Friedrich, L. Chi, Small 8, (2012) 488-503.

5. L. Landau, B. Levich, Acta Physicochem. USSR 17, (1942) $42-54$

6. D. Quéré, A. de Ryck, Ann. Phys. Fr. 23, (1998) 1-149

7. A.S. Dimitrov, K. Nagayama, Langmuir 12, (1996) 13031311.

8. G. Berteloot, C.-T. Pham, A. Daerr, F. Lequeux L. Limat, Europhys. lett. 83,(2008) 14003

9. M. Le Berre, Y. Chen, D. Baigl, Langmuir 25, (2009) 25542557.

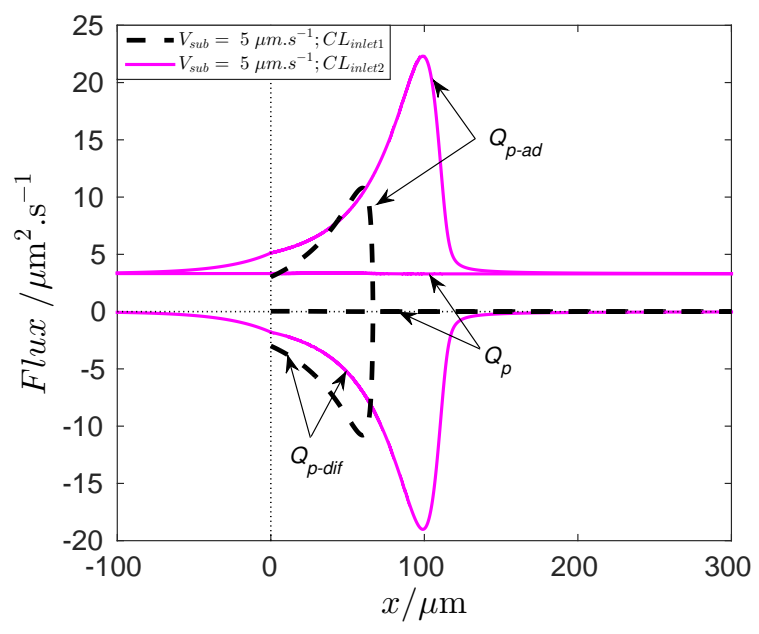

Fig. 10. Diffusion and advection components of the polymer flux for $V_{\text {sub }}=5 \mu \mathrm{m} / \mathrm{s}\left(\phi_{0}=0.01, \mathcal{M}_{G 2 D}\right.$ with $T=25^{\circ} \mathrm{C}$, zoom in the range $-100 \mu \mathrm{m} \leq x \leq 300 \mu \mathrm{m})$.

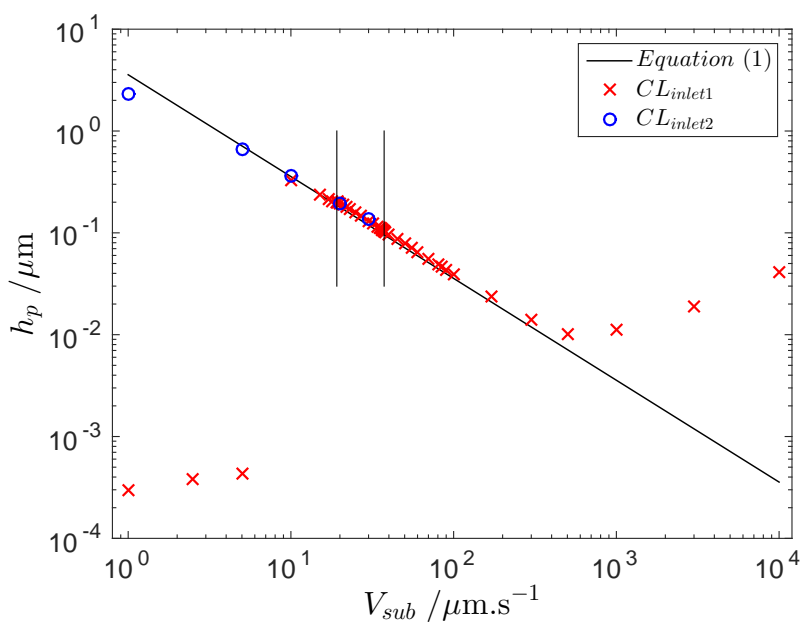

Fig. 11. Dry-deposit thickness as a function of substrate velocity $\left(\mathcal{M}_{G 2 D}\right.$ model with $T=25^{\circ} \mathrm{C}$ and $\left.\phi_{0}=0.01\right)$. The vertical bars delimit the periodic regime, where the deposit thickness is averaged over a cycle. The minimum at $V_{\text {sub }}=500 \mu \mathrm{m} . \mathrm{s}^{-1}$ corresponds to the transition between the evaporative regime and the Landau-Levich regime.

10. G. Jing G, H. Bodiguel H., F. Doumenc, B. Guerrier, Langmuir 26, (2010) 2288-2293.

11. D. Grosso, J. Mat. Chem. 21, (2011) 17033

12. G. Berteloot, A. Daerr, F. Lequeux L. Limat, Chem. Eng. Proc.: Proc. Int. 68, (2013) 69-73

13. L. Frastia, A.J. Archer, U. Thiele, Soft Matter 8, (2012) 11363-11386

14. M. R. E. Warner, R. V. Craster, O.K. Matar, J. Colloid Interface Sci. 267, (2003) 92-110

15. L. Frastia A. J. Archer, U. Thiele, Phys. Rev. Lett. 106, (2011) 077801

16. R. V. Craster, O. K. Matar, K. Sefiane, Langmuir 25, (2009) 3601

17. M. H. Köpf, S. V. Gurevich, R. Friedrich, L. Chi, Langmuir 26, (2010) 10444-10447. 
18. F. Doumenc, B. Guerrier, Europhys. Lett. 103, (2013) 14001.

19. A. Oron, S.H. Davis, S.G. Bankoff, Rev. Mod. Phys. 69, (1997) 931-980.

20. R. Ober, L. Paz, C. Taupin, P. Pincus, S. Boileau, Macromolecules 16, (1983) 50-55.

21. P.G. de Gennes, Rev. Mod. Phys., 57, (1985) 827-863. 1985.

22. V. M. Starov, M. G. Velarde, J. Phys.- Condens. Matter, 21 (2009) 464121.

23. J.M. Zilinski, Macromol. 29, (1996) 6044.

24. U. Thiele, D. V. Todorova, H. Lopez, Phys. Rev. Lett., 111, (2013) 117801.

25. P.J. Flory, Principles of polymer chemistry (Cornell University Press, Ithaca, NY, 1953)

26. J. Eggers and L.M. Pismen, Phys. Fluids, 22, (2010) 112101.

27. G. Barnes, Colloid Interface Sci. 25, (1986) 89-200.

28. F. Doumenc, B. Guerrier, Langmuir 26, (2010) 1395913967.

29. J.E. Mark, Polymer data Handbook (Oxford University Press, New-York, 1999).

30. Y. Marcus, The properties of solvents (John Wiley and sons, Chichester, 1998).

31. F. Doumenc, B. Guerrier B., C. Allain, J. Chem. Eng. Data 50, (2005) 983-988.

32. R. D. Deegan, O. Bakajin, T. F. Dupont, G. Huber, S. R. Nagel, T. A. Witten, Nature 389, (1997) 827-829.

33. C. Poulard, P. Damman, Europhys. lett., 80 (2007) 64001.

34. A.M. Cazabat, P. Carles, MRS Proceedings 248, (1991) 519.

35. M. H. Kőpf, U. Thiele, Nonlinearity, 27 (2014) 2711-2734. 